\title{
EFEITO DO PROCESSAMENTO DE DIETAS COM DIFERENTES NÍVEIS DE CARBOIDRATOS E LIPIDEOS SOBRE A COMPOSIÇÃO CORPORAL E PERFIL DE ÁCIDOS GRAXOS DO FILÉ DO PACU (Piaractus mesopotamicus)
}

\author{
Claucia Aparecida Honorato ${ }^{1}$, Cleujosí da Silva Nunes ${ }^{2}$, Elma NeIDE VASCONCElos \\ MARTINS CARRILHO ${ }^{3}$, GILBERTO MORAES ${ }^{3}$ \\ ${ }^{1}$ Professora Doutora do Centro Universitário da Grande Dourados, Dourados, MS, Brasil. honoratocl@ yahoo.com.br \\ ${ }^{2}$ Pós-graduanda da Universidade Federal de São Carlos, São Carlos, SP, Brasil. \\ ${ }^{3}$ Professores Doutores da Universidade Federal de São Carlos, São Carlos, SP, Brasil.
}

O objetivo deste trabalho foi avaliar o efeito da extrusão e da peletização de dietas contendo dois níveis de carboidrato (40 e 50\%) e dois níveis de lipídeos (4 e 8\%) sobre o fator de condição, composição corporal e o perfil de ácidos graxos de Piaractus mesopotamicus. Foram produzidas oito dietas isoprotéicas $(26,7 \pm 0,7 \% \quad \mathrm{~PB})$ fornecidas por um período de 90 dias ad libtum. Os parâmetros avaliados foram: fator de condição, composição corporal e perfil de ácidos graxos do filé. O fator de condição mostrou respostas diferentes de acordo com o tipo de processamento. A composição corporal dos pacus não revelou diferença estatística. As dietas peletizadas com $40 \%$ de carboidrato apresentaram a maior relação entre n3/n6 em comparação às dietas extrusadas. O perfil de ácidos graxos do filé de pacu sofreu grande influência da interação dos carboidratos, lipídeos e processamento. $\mathrm{O}$ aumento de carboidrato em dietas extrusadas produziu pacus com maiores teores de ômega3.

PALAVRAS-CHAVE: Composição de ácidos graxos; pacu; processamento de dieta.

\author{
EFFECT OF PROCESSING OF DIETS WITH DIFFERENT LEVELS OF \\ CARBOHYDRATES AND LIPIDS ON BODY COMPOSITION AND FATTY ACID \\ COMPOSITION OF FILET OF Piaractus mesopotamicus
}

\begin{abstract}
The objective of this study was to evaluate the effect of extruded or pelletized diets on the condition factor, body composition and fatty acid composition of Piaractus mesopotamicus. Eight isoproteic diets $\left(220 \mathrm{~g} \cdot \mathrm{kg}^{-1}\right.$ digestible protein) were supplied for 90 days ad libtum. The following parameters were evaluated: condition factor, body composition, and fatty acid composition. The condition factor showed different responses according to the type of processing. The body composition of the fish
\end{abstract}

submitted to different diets showed no statistical difference. The pellet diets with $40 \%$ carbohydrate consistently showed the highest ratio compared to $n 3 / n 6$ extruded diets. The fatty acid composition of the fillet showed an interaction of carbohydrate, lipids and processing. The increase in carbohydrate in the extruded diets produced Piaractus mesopotamicus with higher levels of omega-3. 


\section{INTRODUÇÃO}

O pacu, Piaractus mesopotamicus (Holmberg, 1887), é comercialmente interessante devido à sua adaptação ao cativeiro, tecnologia de reprodução conhecida, boa aceitação de ração e carne saborosa para consumo. Entretanto, o pacu é classificado como pescado gordo, pois sua composição corporal contém alto teor de gordura, com cerca de 8 a $10 \%$ de lipídeos na carcaça (STECH, 1999; VIEGAS et al., 2008).

O excesso de gordura na carcaça não é uma característica desejável, devendo-se manter níveis que não afetem as características organolépticas da carne e o rendimento de filé, para não comprometer o valor comercial do peixe (MEURER et al., 2002). A fração lipídica dos peixes apresenta alto teor em ácidos graxos insaturados (AGI), que a predispõem à rancidez oxidativa. Os ácidos graxos (AG) dos peixes de água doce são provenientes dos nutrientes ingeridos na dieta e das modificações fisiológicas (HUANG et al., 1998), pois algumas espécies são capazes de alongar e dessaturar AG de cadeia curta em AG de cadeia longa de interesse nutricional, como os ácidos eicosapentaenóico (EPA) e o docosahexaenóico (DHA) (BELL et al., 1986).

Modificações qualitativas e quantitativas da ração podem alterar a composição corporal dos peixes (AIURA \& CARVALHO, 2004; FUJIMOTO et al., 2007; VIEGAS et al., 2008), assim como o processamento das dietas, que disponibiliza nutrientes de formas diferentes ao melhorar a digestibilidade, levando à maior deposição de gordura na carcaça do Piaractus mesopotamicus (HONORATO et al., 2007).

Entre os processamentos das dietas mais utilizados na aquicultura estão a extrusão e a peletização, os quais propiciam modificações benéficas do amido cru, refletindo em aumento no aproveitamento nutricional das rações pelos peixes (HONORATO et al., 2007). Frente a esse cenário de processamento de dietas, as modificações e as interações ocorridas entre os ingredientes durante o processamento da dieta podem refletir no aproveitamento alimentar.
Espécies de peixes como Sparus aurata, Oncorhynchus mykiss e Salmo solar apresentam baixa capacidade para aproveitar os amidos crus das dietas, quando se compara com o amido cozido ou gelatinizado, atribuindo a estes melhores coeficientes de digestibilidade e disponibilidade da energia das dietas (CHENG \& HARDY, 2003; VENOU et al., 2003; YOUNG et al., 2006), podendo, dessa forma, alterar o metabolismo lipídico e até mesmo o perfil de AG dos peixes.

Este estudo teve como objetivo avaliar o efeito da extrusão e da peletização de dietas contendo dois níveis de carboidrato (40 e 50\%) e dois níveis de lipídeo (4 e 8\%) sobre o fator de condição, composição corporal e o perfil de ácidos graxos do filé de Piaractus mesopotamicus.

\section{MATERIAL E MÉTODOS}

Foram produzidas oito dietas isoprotéicas $(26,7 \pm 0,7 \% \quad \mathrm{~PB})$, contendo dois níveis de carboidratos $(40$ e $50 \%)$ e dois níveis de lipídeos (4\% e $8 \%$ ), quatro delas submetidas ao processo de peletização e quatro ao processo de extrusão (Tabela 1).

O processamento de extrusão foi realizado em equipamento Extrutec de rosca simples, com capacidade nominal de $10 \mathrm{Kg} / \mathrm{h}$. O processamento por peletização ocorreu em peletizadora modelo de laboratório (Califórnia Pellet Mill). Os peletes foram confeccionados com diâmetro entre 1,2 e $1,5 \mathrm{~mm}$ e comprimento entre 1,5 e $3,0 \mathrm{~mm}$. Os ingredientes utilizados foram analisados quanto à sua composição bromatológica (A.O.A.C., 2000).

Foram utilizados 240 alevinos de pacu com peso de $1,17 \pm 0,05 \mathrm{~g}$. Os peixes foram distribuídos em 24 aquários de $150 \mathrm{~L}$ em sistema com abastecimento contínuo de água e aeração constante por meio de compressor radial, com renovação de 20 vezes por dia. A temperatura (29 $\left.{ }^{\circ} \mathrm{C}\right)$ e o oxigênio dissolvido $(5,6 \pm 0,42 \mathrm{mg} / \mathrm{L}$. $)$ foram monitorados diariamente; o $\mathrm{pH}(7,4 \pm 0,1)$, a alcalinidade $(5,6 \pm 0,42 \mathrm{mg} / \mathrm{L})$ e a condutividade $(190,5 \pm 1,5 \mu \mathrm{S} / \mathrm{cm})$, semanalmente. 
Tabela 1. Composição bromatológica das dietas experimentais

\begin{tabular}{|c|c|c|c|c|}
\hline \multirow{2}{*}{$\begin{array}{l}\text { Carboidrato (\%) } \\
\text { Lipídeo }(\%)\end{array}$} & \multicolumn{2}{|c|}{40} & \multicolumn{2}{|c|}{50} \\
\hline & 4 & 8 & 4 & 8 \\
\hline \multicolumn{5}{|l|}{ Ingredientes (\%) } \\
\hline Farinha de peixe ${ }^{\mathrm{A}}$ & 10,4 & 10,4 & 10,4 & 10,4 \\
\hline Farelo de soja ${ }^{\mathrm{B}}$ & 31 & 13 & 25,5 & 11,5 \\
\hline Soja integral tostada ${ }^{\mathrm{C}}$ & 4,7 & 22 & 7,5 & 20,8 \\
\hline Farelo de milho & 26,9 & 29,9 & 25,5 & 25 \\
\hline Amido de milho & -- & -- & 8,9 & 12,5 \\
\hline Farelo de trigo & -- & 2 & 9 & 8 \\
\hline Farinha de trigo & 6,5 & 8 & 2,6 & 2 \\
\hline Quirera de arroz & 8,5 & 7 & 8 & 7,9 \\
\hline Óleo de soja & 0,5 & 0,4 & -- & 0,9 \\
\hline Celulose microfina ${ }^{\mathrm{D}}$ & 10,5 & 6,3 & 1,6 & -- \\
\hline Mistura mineral - vitamínico* & 1 & 1 & 1 & 1 \\
\hline \multicolumn{5}{|l|}{ Composição química analisada } \\
\hline Matéria seca & 89,5 & 89,5 & 88,7 & 89,7 \\
\hline Proteína bruta & 27,0 & 27,5 & 26,1 & 25,7 \\
\hline Lipídeo & 4,0 & 8,0 & 4,1 & 8,0 \\
\hline Matéria mineral & 5,2 & 5,4 & 5,3 & 5,0 \\
\hline Carboidrato** & 40,1 & 40,0 & 50,0 & 49,2 \\
\hline Fibra bruta & 13,1 & 8,6 & 3,9 & 1,9 \\
\hline Energia bruta (kcal.kg-1) & 3698,4 & 4023,8 & 4022,3 & 4261,6 \\
\hline \multicolumn{5}{|l|}{ Perfil de AG analisado } \\
\hline$\sum \square \mathrm{n} 3$ & 5,1 & 7,1 & 4,4 & 4,1 \\
\hline$\sum \square \mathrm{n} 6$ & 44,1 & 51,3 & 44,1 & 45,5 \\
\hline Ácidos graxos saturados & 44,0 & 37,3 & 49,2 & 48,5 \\
\hline Ácidos graxos monoinsaturados & 6,5 & 4,1 & 2,2 & 1,9 \\
\hline Ácidos graxos poliinsaturados & 49,5 & 58,6 & 48,6 & 49,6 \\
\hline Ácidos graxos insaturados & 56,0 & 62,7 & 50,8 & 51,5 \\
\hline $\mathrm{n} 3 / \mathrm{n} 6$ & 0,12 & 0,14 & 0,10 & 0,09 \\
\hline \multicolumn{5}{|c|}{ 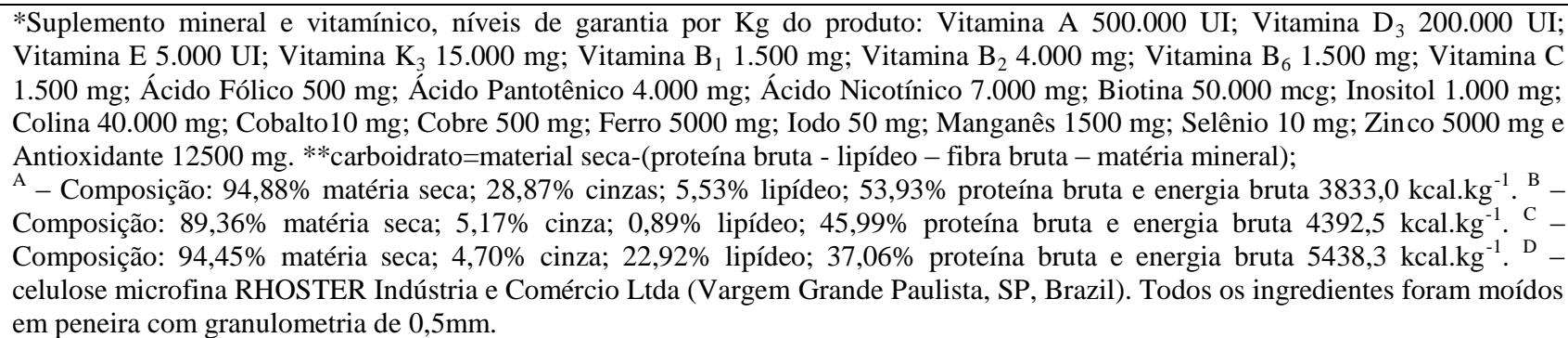 } \\
\hline
\end{tabular}

Os peixes foram alimentados por 90 dias até a saciedade, duas vezes ao dia. Ao final desse período, os peixes passaram por jejum de 48 horas para o esvaziamento gástrico e, logo após, foram anestesiados (por meio de imersão em gelo). Os peixes foram pesados e medidos para-se avaliar o valor do intercepto da relação peso e comprimento (K) denominado Fator de condição(k) = peso /comprimento ${ }^{\mathrm{b}}$, sendo $b$ obtido através da equação alométrica da relação peso / comprimento $\left(\mathrm{y}=\mathrm{ax} \mathrm{x}^{\mathrm{b}}\right)$.
Posteriormente, foram coletados três exemplares de cada repetição para a composição corporal e três exemplares para as análises de perfil cromatográfico do filé.

No início do ensaio de crescimento, foram abatidos dez alevinos (não pertencentes aos tratamentos) e, ao final do período experimental, três exemplares de cada repetição, que foram usados para obtenção das amostras da determinação de composição corporal. O material foi previamente 
moído e seco em estufa de circulação de ar a $65^{\circ} \mathrm{C}$ até peso constante. Na caracterização bromatológica da carcaça, foram avaliados os teores de matéria seca, proteína bruta, lipídeos (A.O.A.C., 2000) e de energia bruta.

Para a analise de perfil cromatográfico, os filés dos peixes foram retirados. As amostras de filés de pacu foram moídos e liofilizados (Savant Modulate - Freezer Dryer) para determinação de lipídeos totais (LT) (BLIGH \& DYER, 1959). A transesterificação dos lipídeos dos filés foi obtida por processo de metilação (5509 da ISO, 1978).

Os AG das dietas e dos filés foram determinados por meio de cromatografia de gás (Shimadzu, modelo GC - 14B), com detector de ionização de chama e coluna capilar de polietilenoglicol com dimensões de $30 \mathrm{~m} \times 0,25 \mathrm{~mm}$, $0,25 \mu \mathrm{m}$ (Omegawax 250). O volume de injeção da amostra foi $0,5 \mu \mathrm{L}$. O aquecimento da coluna seguiu a programação: $100^{\circ} \mathrm{C}$ por 2 minutos, com rampa de temperatura de $4^{\circ} \mathrm{C} / \mathrm{min}$ até $240^{\circ} \mathrm{C}$. As temperaturas do injetor e detector foram $250^{\circ} \mathrm{C}$ e $280^{\circ} \mathrm{C}$, respectivamente. As curvas analíticas de calibração foram obtidas com solução-padrão mista de ésteres metílicos dos AG investigados (SUPELCO, 37 components FAME mix). A quantificação dos AG foi realizada após a identificação dos picos obtidos através de comparação entre os tempos de retenção das amostras e os padrões.

Os resultados foram analisados segundo um delineamento inteiramente casualizado (DIC), com oito tratamentos em esquema fatorial $2 \times 2 \times 2$, constituído de dois níveis de carboidrato (40 e $50 \%$ CHO), dois níveis de lipídeo (4 e $8 \%$ EE) e dois processamentos das dietas (peletização e extrusão), com três repetições. Os dados foram analisados de acordo com teste paramétrico ANOVA, seguido de pós-teste de Tukey, admitindo-se $\mathrm{P}<0,05$ como nível de significância. $O$ programa estatístico utilizado para as comparações das médias foi o Statistical Analysis System (SAS Intitule Inc., version 6.12, 1999).

\section{RESULTADOS}

O fator de condição revelou respostas diferentes de acordo com o tipo de processamento. Nas dietas peletizadas com $40 \%$ de carboidrato, independente do nível de lipídeo, apresentaram fator de condição superior aos peixes alimentados com dietas extrusadas. Nas dietas com $50 \%$ de carboidrato, $\mathrm{o}$ processamento não diferiu significativamente (Figura 1).

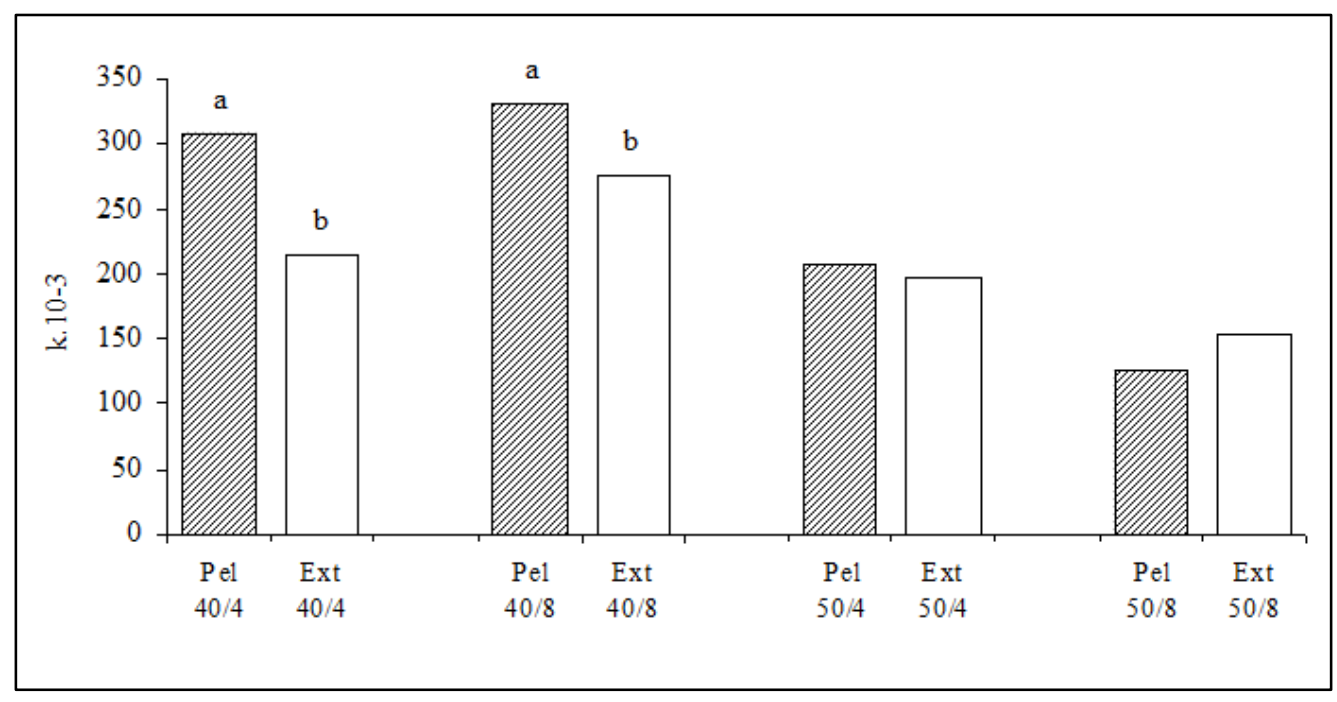

Figura 1. Fator de condição de pacu submetido a dietas peletizadas ou extrusadas com diferentes níveis de carboidrato e lipídeo. Letras distintas (comparação entre processamento - peletizadas ou extrusadas) reportam diferença significativa pelo teste de Tukey $(\mathrm{P}<0,05)$. Valores expressos em média $(\mathrm{n}=30)$. (Pelpeletizado, Ext extrusado, 40 e 50 - níveis de carboidratos; 4 e 8 - níveis de lipídeos.

Os valores médios de composição corporal dos pacus após alimentação com diferentes dietas não apresentaram diferença (Tabela 2). Observou-se desenvolvimento de composição corporal dos peixes, uma vez que, numericamente, os teores de lipídeos e energia bruta corporais aumentaram quando comparados os dados do início e os do final do período de alimentação 
Tabela 2. Valores de composição corporal de alevinos de pacu

\begin{tabular}{|c|c|c|c|c|c|c|}
\hline \multirow{2}{*}{\multicolumn{3}{|c|}{ Carcaça }} & Umidade & $\begin{array}{c}\text { Proteína } \\
\text { Bruta }\end{array}$ & Lipídeos & $\begin{array}{c}\text { Energia } \\
\text { bruta }\end{array}$ \\
\hline & & & $\%$ & $\%$ & $\%$ & Kcal.kg_1 \\
\hline \multicolumn{3}{|c|}{ Composição inicial } & $67,63 \pm 1,7$ & $42,34 \pm 2,5$ & $9,62 \pm 0,6$ & $2817,19 \pm 27,3$ \\
\hline \multicolumn{7}{|c|}{ Composição Final } \\
\hline $\begin{array}{l}\text { Lipídeo } \\
(\%)\end{array}$ & $\begin{array}{l}\text { Carboidrato } \\
(\%)\end{array}$ & Processamento & & & & \\
\hline 4 & 40 & Peletizado & $66,85 \pm 0,5$ & $32,62 \pm 3,3$ & $16,29 \pm 2,4$ & $3638,27 \pm 123,2$ \\
\hline 4 & 50 & Peletizado & $69,84 \pm 3,2$ & $36,25 \pm 2,5$ & $23,83 \pm 1,0$ & $3642,52 \pm 142,9$ \\
\hline 4 & 40 & Extrusado & $68,03 \pm 1,8$ & $32,31 \pm 2,4$ & $18,72 \pm 1,8$ & $4386,92 \pm 16,9$ \\
\hline 4 & 50 & Extrusado & $67,84 \pm 0,5$ & $31,17 \pm 4,8$ & $22,42 \pm 3,3$ & $3702,84 \pm 304,1$ \\
\hline 8 & 40 & Peletizado & $68,19 \pm 0,7$ & $32,47 \pm 1,6$ & $21,98 \pm 2,5$ & $3719,01 \pm 223,4$ \\
\hline 8 & 50 & Peletizado & $70,49 \pm 2,8$ & $36,69 \pm 1,1$ & $26,44 \pm 3,3$ & $3643,93 \pm 16,1$ \\
\hline 8 & 40 & Extrusado & $67,98 \pm 0,7$ & $33,22 \pm 3,6$ & $20,02 \pm 2,4$ & $4025,67 \pm 58,0$ \\
\hline 8 & 50 & Extrusado & $69,25 \pm 3,8$ & $34,51 \pm 3,7$ & $25,93 \pm 2,9$ & $3600,43 \pm 70,7$ \\
\hline
\end{tabular}

As variações impostas às dietas e suas relações afetaram $(\mathrm{P}<0,01)$ o perfil de $A G$ do filé de pacu. A interação entre níveis de lipídeos e níveis de carboidratos influenciou $(\mathrm{P}<0,01)$ a quantidade de lipídeos totais (LT), somatória de AG n3 ( $\left.\sum \mathrm{n} 3\right)$, AG monoinsaturados (AGM) e AG polinsaturados (AGP). A interação entre nível de carboidratos e processamento $(\mathrm{P}<0,01)$ foi expressa em $\mathrm{AG} \sum \mathrm{n} 3$, AG $\sum n 6, \quad A G$ saturados (AGS), AGM e AG insaturados (AGI). Houve interação $(\mathrm{P}<0,01)$ dos níveis de lipídeos e processamento em todas as variáveis do perfil de AG do filé (Tabela 3).

Tabela 3. Perfil de ácidos graxos do filé do pacu

\begin{tabular}{|c|c|c|c|c|c|c|c|c|c|}
\hline $\begin{array}{r}\text { Níveis nc } \\
(\%\end{array}$ & $\begin{array}{l}\text { as dietas } \\
\text { \%) }\end{array}$ & $\begin{array}{l}\text { Processa- } \\
\text { mento }\end{array}$ & & & & Ácidos graxo & $(\%)$ & & \\
\hline $\begin{array}{l}\text { Carbo- } \\
\text { idrato }\end{array}$ & Lipídeo & & $\begin{array}{l}\text { Lipídeos } \\
\text { totais }\end{array}$ & $\begin{array}{l}\text { Somatória de } \\
\text { ácidos graxos } \\
\text { n3 }\end{array}$ & $\begin{array}{c}\text { Somatória de } \\
\text { ácidos graxos } \\
\text { n6 }\end{array}$ & $\begin{array}{l}\text { Ácidos graxos } \\
\text { saturados }\end{array}$ & $\begin{array}{l}\text { Ácidos graxos } \\
\text { mono- } \\
\text { insaturados }\end{array}$ & $\begin{array}{c}\text { Ácidos graxos } \\
\text { poli- } \\
\text { insaturados }\end{array}$ & $\begin{array}{c}\text { Ácidos graxos } \\
\text { insaturados }\end{array}$ \\
\hline Efeito da & ss médias & & & & & & & & \\
\hline 40 & & & $9,28 \pm 3,0$ & $4,56 \pm 0,5$ & $23,88 \pm 4,1$ & $57,55 \pm 3,5$ & $12,76 \pm 1,1$ & $29,20 \pm 4,1$ & $42,36 \pm 3,5$ \\
\hline 50 & & & $10,19 \pm 1,9$ & $4,27 \pm 1,0$ & $18,86 \pm 3,0$ & $60,82 \pm 2,8$ & $17,13 \pm 2,4$ & $22,04 \pm 3,5$ & $38,01 \pm 2,8$ \\
\hline & 4 & & $7,67 \pm 1,8$ & $4,08 \pm 0,5$ & $18,89 \pm 3,5$ & $61,41 \pm 2,9$ & $15,45 \pm 2,2$ & $22,66 \pm 4,4$ & $37,35 \pm 2,8$ \\
\hline & 8 & & $11,79 \pm 1,0$ & $4,74 \pm 1,0$ & $23,85 \pm 3,8$ & $56,96 \pm 2,3$ & $14,44 \pm 3,4$ & $28,58 \pm 4,6$ & $43,02 \pm 2,3$ \\
\hline & & $\begin{array}{l}\text { Peletiza- } \\
\text { ção }\end{array}$ & $9,52 \pm 1,8$ & $4,34 \pm 0,8$ & $20,26 \pm 3,5$ & $59,83 \pm 4,1$ & $16,04 \pm 3,3$ & $24,09 \pm 5,6$ & $39,66 \pm 4,1$ \\
\hline & & Extrusão & $9,95 \pm 3,1$ & $4,48 \pm 0,9$ & $22,49 \pm 5,2$ & $58,54 \pm 3,1$ & $13,85 \pm 2,2$ & $27,15 \pm 5,1$ & $40,71 \pm 3,0$ \\
\hline F - valore & es (NOV & & & & & & & & \\
\hline Níveis de & e carboid & Iratos & $6,94^{*}$ & $61,98^{* *}$ & $16115,67^{* *}$ & $12452,51^{* *}$ & $241776,31^{* * *}$ & $23522,46^{* *}$ & $27,29^{* *}$ \\
\hline Níveis de & e lipídeo & & $142,43^{* *}$ & $312,00^{* *}$ & $15680,13^{* *}$ & $23108,26^{\text {** }}$ & $12800,56^{\text {** }}$ & $16093,93^{* *}$ & $46,23^{* *}$ \\
\hline Processar & mentos & & $1,56^{\mathrm{ns}}$ & $15,94^{* *}$ & $3184,13^{* *}$ & $1967,06^{* *}$ & $60859,26^{\text {*** }}$ & $4288,30^{* *}$ & $1,59^{\mathrm{ns}}$ \\
\hline Carboidr & atos x Li & ipídeos & $8,89^{*}$ & $123,68^{* *}$ & $1,24^{\mathrm{ns}}$ & $52,20^{* *}$ & $213,47^{\text {*** }}$ & $137,31^{\text {** }}$ & $1,16^{\mathrm{ns}}$ \\
\hline $\begin{array}{l}\text { Carboidr } \\
\text { Processar }\end{array}$ & $\begin{array}{l}\text { atos } \\
\text { mentos }\end{array}$ & $\mathrm{x}$ & $0,26^{\mathrm{ns}}$ & $806,65^{* *}$ & $5670,19^{* *}$ & $3655,63^{* *}$ & $21732,24^{* * *}$ & $1,14^{\mathrm{ns}}$ & $5,12^{*}$ \\
\hline Lipídeos & $\mathrm{x}$ Proce & ssamentos & $13,26^{* *}$ & $110,98^{* *}$ & $414,85^{* *}$ & $7215,76^{* *}$ & $21020,21^{\text {** }}$ & $306,44^{* *}$ & $7,59^{*}$ \\
\hline $\begin{array}{l}\text { Carboidr } \\
\text { Processar }\end{array}$ & $\begin{array}{l}\text { atos x I } \\
\text { mentos }\end{array}$ & ipídeos x & $38,09^{* *}$ & $264,55^{* *}$ & $652,78^{* *}$ & $0,72^{\mathrm{ns}}$ & $10383,71^{* *}$ & $106,59^{* *}$ & $0,01^{\mathrm{ns}}$ \\
\hline
\end{tabular}

Valores expressos em média \pm D.P. de $n=9$ peixes; Letras maiúsculas nas colunas indicam diferença significativa pelo teste de Tukey a $5 \%$ de probabilidade; $* *(\mathrm{P}<0,01) ; *(\mathrm{P}<0,05) ;{ }^{\text {ns }}$ (não significativo). 
A interação entre os níveis de lipídeos e níveis de carboidratos revelou que o aumento de carboidratos e o aumento de lipídeos nas dietas levaram ao acréscimo de LT no filé. O aumento de lipídeo na dieta elevou a quantidade de AG $\sum \mathrm{n} 3$ e
AGP e diminuição nos AGS e AGM. O aumento de carboidrato elevou as médias de AGS e AGM, já nas dietas com alto lipídeo resultou em elevação de AG $\sum$ n3 e diminuição de AGP (Tabela 4).

Tabela 4. Interação de níveis de carboidratos e lipídeos no perfil de ácidos graxos no filé de pacu

\begin{tabular}{|c|c|c|}
\hline \multirow[b]{2}{*}{ Níveis de lipídeo (\%) } & \multicolumn{2}{|c|}{ Nível de carboidrato (\%) } \\
\hline & 40 & 50 \\
\hline & \multicolumn{2}{|c|}{ Lipídeos totais do filé (\%) } \\
\hline 4 & $6,70 \pm 2,5^{\mathrm{Bb}}$ & $8,64 \pm 0,2^{\mathrm{Ba}}$ \\
\hline \multirow[t]{2}{*}{8} & $11,85 \pm 1,2^{\mathrm{Aa}}$ & $11,73 \pm 1,0^{\mathrm{Aa}}$ \\
\hline & \multicolumn{2}{|c|}{ Somatória de ácidos graxos n 3 (\%) } \\
\hline 4 & $4,43 \pm 0,5^{\mathrm{Ba}}$ & $3,73 \pm 0,2^{\mathrm{Bb}}$ \\
\hline \multirow{2}{*}{8} & $4,67 \pm 0,8 \mathrm{Ab}$ & $4,79 \pm 1,5$ \\
\hline & \multicolumn{2}{|c|}{ Ácidos graxos saturados (\%) } \\
\hline 4 & $59,88 \pm 3,9^{\mathrm{Ab}}$ & $62,93 \pm 1,4^{\mathrm{Aa}}$ \\
\hline \multirow[t]{2}{*}{8} & $55,22 \pm 0,4$ & $58,69 \pm 2,1$ \\
\hline & \multicolumn{2}{|c|}{ Ácidos graxos monoinsaturados (\%) } \\
\hline 4 & $13,19 \pm 0,7^{\mathrm{Ab}}$ & $17.69 \pm 0.9^{\mathrm{Aa}}$ \\
\hline \multirow[t]{2}{*}{8} & $12,31 \pm 0,9^{\mathrm{Bb}}$ & $16,56 \pm 4,0$ \\
\hline & \multicolumn{2}{|c|}{ Ácidos graxos poliinsaturados (\%) } \\
\hline 4 & $25.96+3.0^{\mathrm{Ba}}$ & $1935+2.4^{\mathrm{Ba}}$ \\
\hline 8 & $32,43 \pm 1,3^{\mathrm{Aa}}$ & $24,72 \pm 1,89$ \\
\hline
\end{tabular}

Médias seguidas da mesma letra (maiúscula comparação na coluna, minúscula comparação na linha) não diferem significativamente pelo teste de Tukey a $5 \%$ de probabilidade.

Tabela 5. Interação de níveis de carboidratos e processamentos e níveis de lipídeos e processamentos no perfil de ácidos graxos do filé de pacu

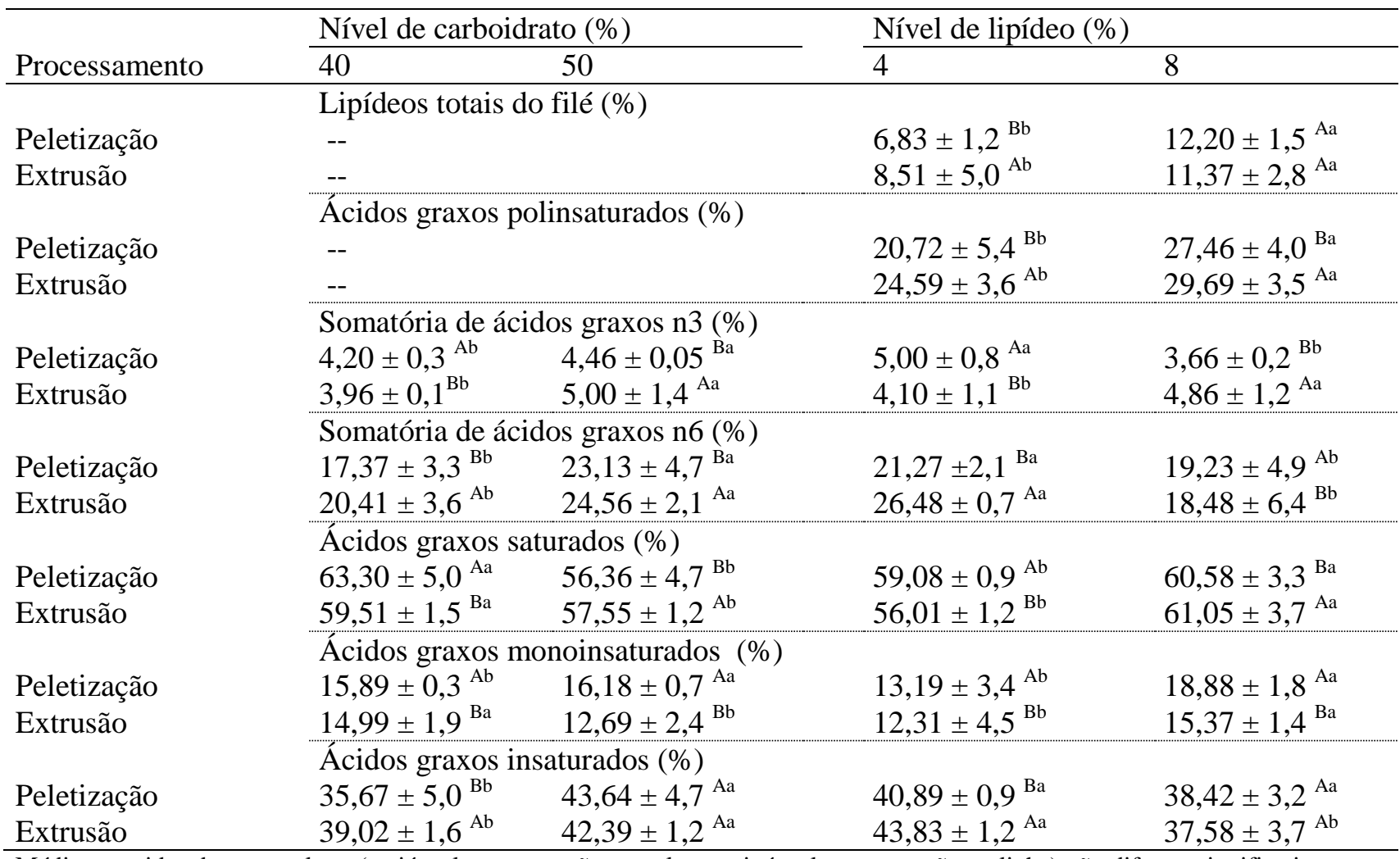

Médias seguidas da mesma letra (maiúscula comparação na coluna, minúscula comparação na linha) não diferem significativamente pelo teste de Tukey a 5\% de probabilidade. (--) Não houve interação para esta variável. 
A interação entre nível de carboidratos e processamentos demonstrou que o aumento de carboidrato elevou os AG $\sum \mathrm{n} 3, \mathrm{AG} \sum \mathrm{n} 6$ e AGI. As dietas extrusadas com alto nível de carboidratos apresentaram maiores valores de AG $\sum \mathrm{n} 3$ e AGI. Os maiores valores de AG $\sum \mathrm{n} 6$ foram observados para dietas extrusadas (Tabela 5).

A interação entre níveis de lipídeos e processamentos revelou que o aumento de lipídeo proporciona elevação de LT, AGS, AGM e AGP. As dietas extrusadas em comparação com as dietas peletizadas apresentaram médias superiores de AGP e AGI e médias inferiores de AGM. Contudo, as dietas extrusadas com baixo lipídeo revelaram aumento de LT e AG $\sum$ n6 e diminuição de AG $\sum$ n3 e AGS e a dieta com alto nível de lipídeo aumentou de AG $\sum$ n3 e AGS e diminuíram AG $\sum$ n6, em comparação as dietas peletizadas (Tabela 5).

As dietas peletizadas com $40 \%$ de carboidrato resultaram na maior relação entre $n 3 / n 6$ no filé de pacu, em comparação às dietas extrusadas. No entanto, a dieta extrusada só mostrou aumento dessa relação nas dietas com alto conteúdo de lipídeo $(8 \%)$ e carboidrato (50\%). A relação n3/n6 aumentou conforme o aumento de carboidrato nas dietas extrusadas e diminuiu nas dietas peletizadas (Figura $2 \mathrm{~A}$ e $2 \mathrm{~B})$.

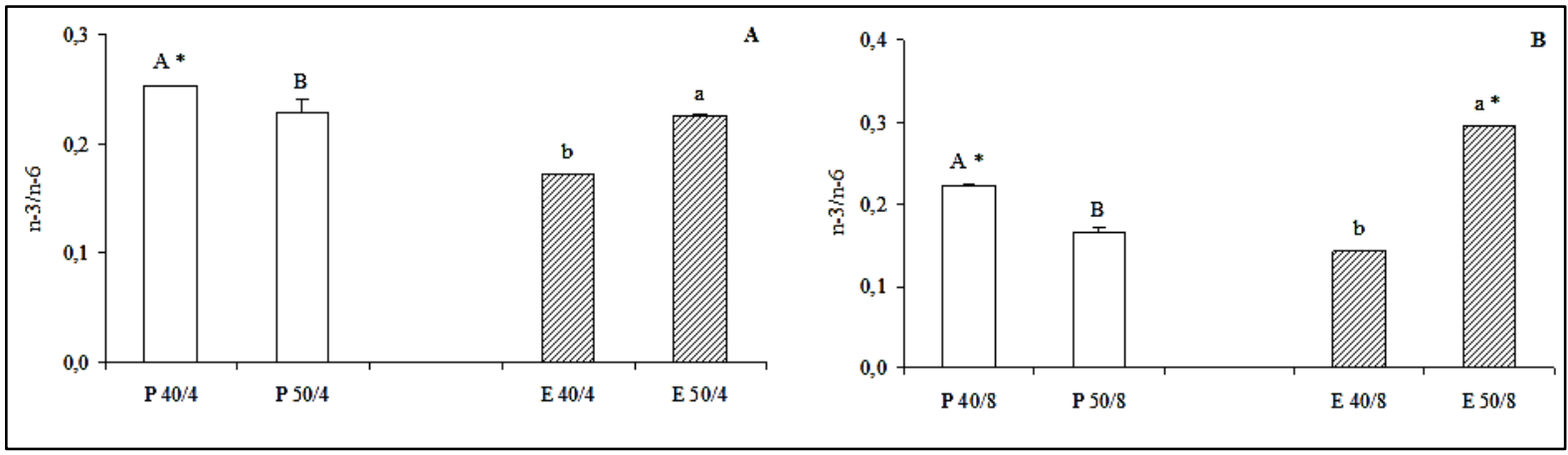

Figura 2 - Relação entre n-3/ n-6 dos filés de pacu submetido ao aumento de carboidrato em dietas (peletizadas ou extrusadas) com (A) baixo e (B) alto nível de lipídeo. Letra diferente (maiúscula comparação entre dietas peletizadas e minúscula comparação entre dietas extrusadas, $\left(^{*}\right)$ comparação entre processamentos).

\section{DISCUSSÃO}

O fator de condição é uma forma indireta de se avaliar o bem estar do peixe por meio da forma de crescimento pela correlação entre peso e comprimento. Segundo WEATHERLEY (1987), é uma forma de se mensurar o grau de atividade alimentar de uma espécie e do aproveitamento da fonte de alimento. $\mathrm{O}$ aumento de carboidrato revelou diminuição do fator de condição, o que pode ser um indicativo de crescimento referente ao acúmulo de gordura nos pacus. No entanto, para $S$. salar alimentados com dietas com níveis crescentes de carboidrato, não foi observada alteração no fator de condição (HEMRE et al., 2002).

Os parâmetros de composição corporal de pacu não diferiram entre os tratamentos. Alguns estudos relatam que a alimentação não altera a composição corporal de peixes, tais como Piaractus mesopotamicus (VIEGAS et al., 2008), Colossoma macropomum (MACEDO-VIEGAS \&
CONTRERAS-GUZMAN, 1998) e Rhamdia quelen (MELO et al., 2002). De forma contrária, a quantidade de proteína muscular de tilápia do Nilo foi alterada pela quantidade de tanino presente na alimentação (AIURA \& CARVALHO, 2004).

O nível de lipídeo e a composição de $A G$ do filé refletem a qualidade do produto que será comercializado. Observou-se que o perfil de AG do filé de pacu foi afetado pela composição nutricional e pelo processamento da dieta. RUNGRUANGSAK-TORRISSEN et al. (2006) descrevem que a qualidade do músculo é um dos fatores preponderantes para a avaliação da qualidade nutricional da dieta.

Nas dietas extrusadas, devido à maior disponibilidade do amido que frequentemente é estocada na forma de gordura, observou-se aumento de lipídeos totais. O aumento de lipídeo nas dietas reflete em regulação do apetite e maior acúmulo de lipídeos na carcaça (YAMAMOTO et al., 2001). O aumento de carboidratos e de lipídeos na dieta de Lates calcarifer reflete em aumento no 
conteúdo de lipídeos na carcaça, o que, consequentemente, diminui a qualidade do produto final (CATACUTAN \& COLOSO et al., 1997).

Como os AG essenciais não são sintetizados "de novo", os AG incorporados nos tecidos representam seu conteúdo nos alimentos ingeridos (FRACALOSSI \& LOVELL, 1995; JOBLING et al., 1998), mas alguns organismos podem fazer síntese de AG a partir de outros nutrientes como carboidratos e proteína, porém, necessitam da enzima dessaturase que insere insaturações entre os carbonos 3-4 e 6-7 da sua porção terminal (ANDRADE \& CARMO, 2006). MARTINO \& TAKAHASHI (2001), em estudo sobre Pseudoplatystoma coruscam, constataram que essa espécie apresenta as enzimas capazes de alongar e dessaturar AG, devido à composição de ácidos graxos da carcaça associada ao tipo de alimentação ofertada. O aumento de AG n-3 foi observado no fígado de Pseudoplatystoma coruscam alimentados com dietas à base de óleo de soja, sugerindo elongamento e desaturação (MARTINO et al., 2002).

Nas dietas extrusadas, tanto o aumento de lipídeo como de carboidratos foi benéfico por proporcionar aumento na quantidade de AGPI (n$3)$ no filé. As concentrações de AGPI n-3 na carne de peixes variam de acordo com a espécie e dependem especialmente da dieta consumida pelo peixe. A maior concentração de AGI nos filés dos peixes alimentados com dietas extrusadas pode ser reflexo da melhora da digestibilidade da fração lipídica especialmente da farinha de peixe (fonte de n-3). O processo de extrusão afeta a composição dos ingredientes e altera os coeficientes de digestibilidade dos nutrientes para Oncorhynchus mykiss, melhorando, em particular, o coeficiente de digestibilidade do lipídeo (CHENG \& HARDY, 2003).

Nutricionalmente, quanto maior o valor de AGP melhor é a qualidade do alimento. Diversos estudos vêm enfatizando que a relação entre $n-3 / n$ 6 fornece indicações importantes, considerando que $n-3$ é benéfico à saúde humana, enquanto $n-6$ não tem a mesma correlação (MAYSER et al., 1998; MARTINO et al., 2002; SUÁREZMAHECHA et al., 2002). A razão entre AG n3/n6 é variável entre as espécies de Brycon (matrinxã, piraputanga e piracanjuba) e observa-se também diferença entre peixes criados em cativeiro e peixes de ambiente natural (MOREIRA et al., 2003). DRUZIAN et al. (2007) observaram para Cyprinus carpio que a alimentação modifica a razão entre n-3/n-6. MOREIRA et al. (2003) observaram que piraputanga (Brycon microlepis) no ambiente natural apresentam relação n-3/n-6 maior que peixes criados em cativeiro.

A relação n-3/n-6 para os tratamentos foi inferior à encontrada para peixes de água doce, a qual varia entre 3,8 a 5,0 (AIURA \& CARVALHO, 2004). No entanto, a relação aproximou-se da obtida para filés de tambaqui (MAIA et al., 1992).

\section{CONCLUSÃO}

$\mathrm{O}$ processamento aplicado à dieta para alimentação do pacu exerceu efeito no perfil de AG do filé e no fator de condição dos peixes. $O$ aumento de carboidrato em dietas extrusadas produziu pacus com maiores teores de $n-3$. Neste contexto, a utilização de dietas extrusadas com $50 \%$ de carboidrato independente do nível de lipídeos (4 ou $8 \%$ ) é apropriada para o pacu.

\section{REFERÊNCIAS}

AIURA, F.S.; CARVALHO, M.R.B. Composição em ácidos graxos e rendimento de filé de tilápia do Nilo (Oreochromis niloticus) alimentada com dietas contendo tanino. Revista Portuguesa de Ciências Veterinárias, v. 99, n. 550, p. 93-98, 2004.

ASSOCIATION OF OFFICIAL ANALYTICAL CHEMISTS. Official methods of analysis of the Association of Official Analytical Chemists. 17th ed. AOAC Inc., Gaithersburg, MD, USA, 2000.

BELL, M.V.; HENDERSON, R.J.; SARGENT, J.R. The role of polyunsaturated fatty acids in fish. Comparative Biochemistry and Physiology, v. 83B, p. 711-719, 1986.

CATACUTAN, M.R.; COLOSO, R.M. Growth of juvenile Asian seabass Lates calcarifer, fed varying carbohydrate and lipd levels. Aquaculture, v. 149, p.137-144, 1997.

CHENG, Z. J.; HARDY, R.W. Effects of extrusion processing of feed ingredients on apparent digestibility coefficients of nutrients for rainbow trout (Oncorhynchus mykiss). Aquaculture Nutrition, v. 9, p. 77-83, 2003.

ANDRADE, P. M. M.; CARMO, M.G. T. Ácidos graxos n-3: um link entre eicosanóides, inflamação e imunidade. Revista de Metabolismo e Nutrição, v.8, n.3, p. 135-143, 2006.

DRUZIAN, J.I.; MARCHESI, C.M.; SCAMPARINI , A.R.P. Perfil de ácidos graxos e composição centesimal de carpas (Cyprinus carpio) alimentadas com ração e com dejetos suínos. Ciência Rural, v. 37, n. 2, p. 539544, 2007.

FRACALOSSI, D.M.; LOVELL, R.T. Growth and polar fatty acid composition of year-1 channel catfish fed various lipid source at two water temperatures. The 
Progressive Fish-Culturist. v.57, p.107-113, 1995.

FUJIMOTO, R. Y.; CASTRO, M.P.; HONORATO, C.A.; NORAES, F.R. Composição corporal e eficiência de utilização de nutrientes por pacus alimentados com ração suplementada com cromo trivalente. Pesquisa Agropecuária Brasileira, v. 42, p. 1763-1768, 2007.

HEMRE， G.-I.; BJØRNEVIK,M.; BEATTIE,C.; BJÖRNSON, B.T.; HANSEN, T. Growth ans saltwather tolerance of juvenile Altantic salmon Salmo salar, reared under different combinations of dietary carbohydrate and photoperid regime. Aquaculture Nutrition, v. 8, p.23-32, 2002.

HONORATO, C.A. ALMEIDA, L.C., Da SILVA NUNES, C. CARNEIRO, D.J. MORAES, G.. Effects of processing on physical characteristics of diets with distinct levels of carbohydrates and lipids: the outcomes on the growth of pacu (Piaractus mesopotamicus). Aquaculture Nutrition, v. 16, p.9199, 2010.

HUANG, C.H., HUANG, M.C., LEE, A.C. Characteristics of lipid peroxidation in sarcoplasmic reticulum of tilapia. Food Science, v. 25, p. 104-108, 1998.

JOBLING, M. KOSKELA, J.; SAVOLAINEN, R. Influence of dietary fat level and increased adiposity on growth and fat deposition in rainbow trout, Oncorhynchus mykiss (Walbaum). Aquaculture Research, v. 29, p. 601-607, 1998.

MAIA, E. L.; RODRIGUEZ-AMAYA, D. B. Fatty acid composition of the total, neutral and phospholipids of the Brazilian freshwater fish Colossoma macropomum. In Food Science and Human Nutrition (G. Charalambous, Ed.), p. 633-642. Elsevier Science, Amsterdam, 1992.

MACEDO-VIEGAS, E.M.; CONTRERAS-GUZMAN, E. Effect of source and levels of dietary lipids on growth, body composition, and fatty acids of the tambaqui (Colossoma macropomum). World Aquaculture, v. 29, n.1, p.66-70, 1998.

MARTINO, R.; TAKAHASHI, N.S. A importância da adição de lipídios em rações para a aquiicultura. Óleos e Grãos, v. 58, p.32-37. 2001.

MARTINO, R.C., CYRINO, J.E.P.; PORTZ, L.; TRUGO, L.C. Performance and fatty acid composition of surubim (Pseudoplatystoma coruscans) fed diets with animal and plant lipids. Aquaculture, v. 209, p.233-246, 2002.

MAYER, P.; MROWIETZ, U.; ARENBERGER, P.; BARTAK, P.; BUCHVALD, J.; CHRISTOPHEERS, E; JABLONSKA, S.; SALMHOFER, W.; SCHILL, W.B; KRAMER, H.J.; SCHLOTZER, E.; MAYER, K; SEEGER, W.; GRIMMINGER, F. Omega-3 fatty acidbased lipid infusion in patients with chronic plaque psoriasis: results of a double-blind, randomized, placebo-controlled, multicender trial. Journal of American Academy Dermatology, v. 38, p. 421, 1998.
MELO, J.F.B., RADUNZ-NETO, J.; SILVA, J.H.S.; TROMBETTA, C.G. Desenvolvimento e composição corporal de alevinos de jundiá (Rhamdia quelen) alimentados com dietas contendo diferentes fontes de lipídeos. Ciência Rural, v. 32, p.323-327, 2002.

MEURER, F., HAYASHI, C., BOSCOLO, W.R., SOARES, C.M. Lipídeos na alimentação de alevinos revertidos de tilápia do Nilo (Oreochromis niloticus). Revista Brasileira de Zootecnia, v. 31, p. 566-573, 2002.

MOREIRA, A.B.; SOUZA, N.E.; VISENTAINER, J.V.; MATSUSHITA, M. Composição de ácidos graxos e teor de lipídios em cabeças de peixes: matrinxã ( $B$. Cephalus), piraputanga (B. Microlepis) e piracanjuba (B. Orbignyanus), criados em diferentes ambientes. Ciência e Tecnologia de Alimentos, v. 32, p. 179-183, 2003.

RUNGRUANGSAK-TORRISSEN， K.; MOSS， R.; ANDRESEN,L.H.; BERG, A.; WAAGBØ. Different expression of typsin and chymostrypsin in relation to growth in Alantic salmon (Salmo salar L.). Fish Physiology and Biochemistry, v. 32, p. 7-23, 2006.

STECH, M.R. ; CARNEIRO, D.J. ; CARVALHO, M.R.B. . Fatores antinutricionais e coeficientes de digestibilidade aparente da proteína de produtos de soja para o pacu (Piaractus mesopotamicus). Acta Scientiarum. Animal Sciences, v. 32, p. 255-262, 2010.

SUÁREZ-MAHECHA, H.; FRANCISCO, A.; BEIRÃO, L.H.; BLOCK, J.M.; SACCOL, A.; PARDOCARRASCO, S. A importância de ácidos graxos poliinsaturados presentes em peixes de cultivo e de ambiente natural para a nutrição humana. Boletim do Instituto de Pesca, v. 28, n.1, p.101-110, 2002.

WEATHERLEY, A.H, GILL, H.S. Growth increases produce by bovine growth hormones in grass pickerel, Esox american vermiculatus (Le Sueur), and the underlying dynamics of muscle fiber growth. Aquaculture, v. 65, p. 55-66, 1987.

YAMAMOTO, T.; KONISHI, K. SHIMA, T.; FURUITA, H.; SUZUKI, N., TABATA, M. Influence in dietary fat and carbohydrate levels on growth and body composition of raibow trout Oncorhynchus mykiss under self- feeding conditions. Fisheries Science, v. 67, p. 221-227, 2001.

VENOU, B., ALEXIS, M. N., FOUNTOULAKI, E., NENGAS, I., APOSTOLOPOULOU, M. \& CASTRITSI-CATHARINOU, I. Effect of extrusion of wheat and corn on gilthead sea bream (Sparus aurata) growth, nutrient utilization efficiency, rates of gastric evacuation and digestive enzyme activities. Aquaculture, v. 225, p. 207-223, 2003.

YOUNG, A., MORRIS, P.C., HUNTINGFORD, F.A. \& SINNOTT, R. Replacing fish oil with pre-extruded carbohydrate in diets for Atlantic salmon, Salmo solar, during their entire marine grow-out phase: Effects on 
growth, composition and colour. Aquaculture, v. 253, p. 531-546, 2006.

VIEGAS, E.M.M.; CARNEIRO, D.J.; URBINATI, E.C.; MALHEIROS, E.B. Farelo de canola em dietas para o pacu Piaractus mesopotamicus (Holmberg 1987): efeitos sobre o crescimento e a composição corporal. Arquivo Brasileiro de Medicina Veterinária e Zootecnia, v.60, n.6, p. 1502-1510, 2008.

Protocolado em: 18 mar. 2011. Aceito em: 26 fev. 2013. 